EYETRACKING Y USABILIDAD:

\title{
CLAVES DE INVESTIGACIÓN EN LOS PROCESOS DE LECTURA EN LÍNEA
}

\author{
EYETRACKING AND USABILITY: \\ KEY RESEARCH IN THE READING ONLINE PROCESS
}

\section{AUTOR}

\section{Fátima Martínez Gutiérrez}

Profesora Asociada. Universidad San Pablo CEU. Madrid (España).

fatima.martinezgutierrez@ceu.es

\section{RESUMEN}

El presente estudio tiene la intención de despejar algunas de las dudas planteadas anteriormente gracias a la información facilitada por fuentes como la página Web del Instituto Poynter (www.poynterextra.org) sobre Eyetracking en Estados Unidos o la obra de Guillermo Franco titulada Cómo escribir para la Web, donde se detalla la labor de investigación ejercida por Nielsen. En esta línea, posteriormente, se especificarán algunas de las conclusiones obtenidas sobre los estudios de Eyetracking llevados a cabo en España y dirigidos por la consultora alt64, empresa especializada en maximizar la rentabilidad de las inversiones en negocios online, que colabora con la Asociación para la Investigación de Medios de Comunicación (AIMC).

\section{PALABRAS CLAVE}

Usabilidad - Eyetracking - Lectura en línea. 


\section{ABSTRACT}

This study aims to dispel some of the questions previously raised by the information provided by sources like the Poynter Institute's web site (www.poynterextra.org) on Eyetracking in the U.S., and the work of Guillermo Franco headed Writing for the Web, which details the research carried out by Nielsen. In this line, then specifying some of the conclusions reached on Eyetracking studies conducted in Spain and led by the consultant Alt64, a company specializing in maximizing the profitability of investments in online business, working with the Partnership for Media Research (AIMC).

\section{KEY WORDS}

Usability - Eyetracking - Read online.

\section{ÍNDICE}

1. Introducción

2. Conceptualización

2.1. Imagen del Eyetracking

3. Terminología relacionada

4. Investigaciones recientes

5. El caso español

6. Críticas versus posibilidades

7. Conclusiones

8. Bibliografía 


\section{Introducción}

“No será mejor leer en una pantalla de cristal líquido, flexible, bien iluminada, con grandes letras y capacidad de enlaces a otros temas a través del hipertexto, que hacerlo en un papel con cara de añoso, mal impreso y lleno de imperfecciones?", se planteaba el Juan Luis Cebrián en la obra El periodista en el burdel. La pretensión de este trabajo consiste en realizar una aproximación a las últimas investigaciones sobre lo que se ha denominado Eyetracking, un término anglosajón cuya traducción equivaldría en castellano al "rastreo del ojo" o "trazabilidad visual".

Entendemos el Eyetracking como una revolucionaria herramienta de análisis, relativamente reciente, que puede servir para averiguar el recorrido que realiza el ojo de una persona cuando se encuentra frente a una página Web de un ordenador. Sin embargo, convendría ser críticos debido a la dificultad que entraña descifrar completamente el comportamiento de un usuario de Internet en líneas generales.

Hasta ahora, se ha estudiado pormenorizadamente qué lugares debe ocupar la publicidad y dónde han de colocarse las noticias más relevantes del día en los diarios de mayor repercusión social. Como es obvio, la portada y las páginas impares se han considerado las más vistas por su ubicación. Asimismo, sucede con los espacios publicitarios y los programas o series que deben ir en el horario de prime time (hora de máxima audiencia), tanto en radio como en televisión. El codiciado minuto de oro no puede desperdiciarse, tan aclamado entre todas las empresas encargadas en la investigación de audiencias y, por supuesto, entre los anunciantes más ambiciosos.

De hecho, el primer estudio independiente de carácter significativo de Eyetracking se desarrolló a finales de la década de los ochenta de la mano del Instituto Poynter, pionero en esta técnica. Poynter trabajó con expertos en la tecnología del Eyetrac 
procedentes de la Gallup Applied Science de Princeton, New Jersey, con el objetivo de estudiar el recorrido visual de los lectores en prensa escrita. Sus resultados fueron publicados en el año 1991 y están recogidos en la obra Eyes on the News. A partir de los primeros hallazgos obtenidos, el Instituto Poynter ha continuado indagando en proyectos de Eyetracking, perfilando sus avances técnicos y centrando sus observaciones en el comportamiento de los internautas en la Red, después del desarrollo de las Tecnologías de la Información y de la Comunicación (TICS) desde la década de los noventa hasta hoy. El mencionado Instituto no ha dejado de proporcionar, desde entonces, datos de interés tanto en el ámbito de la publicidad como en el periodístico e, incluso, en el concerniente al aspecto psicológico.

Jakob Nielsen, gurú de la usabilidad, como le ha dado en llamar Guillermo Franco, autor de la obra Cómo escribir para la Web, se ha convertido en un avezado investigador en esta materia. Sus planteamientos no han dejado indiferentes a quienes pretenden observar los patrones de lecturas que se realizan en las webs de Internet. Nielsen ha llegado a desmitificar muchas teorías que aún hoy para muchos siguen siendo consideradas paradigmáticas. Muestra de algunos de los descubrimientos reside en la siguiente afirmación desprendida del documento oficial del EyeTrack del año 2000:

"¿A dónde van los ojos después de arrancar de la página de inicio de un sitio on line de noticias? Al texto, con mayor probabilidad. No a las fotos o gráficas, como podría esperarse. En su lugar, las breves y leyendas consiguen más fijaciones primero, en general. Los ojos de los lectores on line luego regresan a las fotos y gráficas, algunas veces no hasta que regresen a la primera página después de dar clic sobre un artículo completo".

Si se está incrementando exponencialmente el papel del usuario, debido a su capacidad de interactuar con el entorno digital (persona-ordenador) que le permite 
una mayor interactividad y, por tanto, conversar con el resto de los usuarios, de muchos a muchos, cabe estudiar qué tipo de conversación mantiene con el ordenador y cómo se produce esa interacción. La complejidad del asunto no es baladí, sobre todo, cuando existen tantos interrogantes por responder:

- ¿Qué parámetros de comportamientos suelen regir al usuario? (Número de horas, confianza en el medio digital o actividad continua de los ciudadanos en la red).

- ¿Qué elementos intervienen en la conversación usuario-ordenador? ¿Dónde se localizan los puntos calientes 0 áreas de interés en una Web?

- ¿Qué relación existe entre el Eyetracking y la Usabilidad? ¿Es prioritaria la estética o la funcionalidad en el medio Web?

- ¿Cómo es la lectura en Internet? ¿Lineal y secuencial? ¿No lineal?

- ¿Dónde se lee más en los medios digitales o en los medios impresos?

- ¿Qué ventajas permite la redacción digital?

- ¿Qué función poseen las palabras claves para el Eyetracking?

El presente estudio tiene la intención de despejar algunas de las dudas planteadas anteriormente gracias a la información facilitada por fuentes como la página web del Instituto Poynter (www.poynterextra.org) sobre Eyetracking en Estados Unidos o la obra de Guillermo Franco titulada Cómo escribir para la Web, donde se detalla la labor de investigación ejercida por Nielsen. En esta línea, posteriormente, se especificarán algunas de las conclusiones obtenidas sobre los estudios de Eyetracking llevados a cabo en España y dirigidos por la consultora alt64, empresa especializada en maximizar la rentabilidad de las inversiones en negocios online, que colabora con la Asociación para la Investigación de Medios de Comunicación (AIMC).

Sin embargo, a pesar de las posibilidades de conocimiento que ha permitido esta herramienta, se subrayarán al final del trabajo la problemática que envuelve esta técnica y las discusiones que ha provocado en los ámbitos periodísticos. 


\section{Conceptualización}

La revista multidisciplinar sobre diseño de interacción No solo usabilidad (del 27 de octubre de 2007) afirma que el concepto del Eyetracking hace referencia al

"conjunto de tecnologías que permiten monitorizar y registrar la forma en la que una persona mira una determinada escena o imagen, en concreto en qué áreas fija su atención, durante cuánto tiempo y qué orden sigue su exploración visual".

En definitiva, como también apunta un estudio realizado por Usolab, "el Eyetracking es una tecnología que permite seguir los movimientos oculares de una persona para inferir qué mira y qué ve".

La revista especializada No sólo usabilidad apunta que en este contexto de Interacción Persona-Ordenador (IPO) se distinguen dos posibles vertientes de aplicación del Eyetracking:

- Uso como dispositivo de entrada e interacción

- Uso como herramienta para la evaluación objetiva de interfaces.

Respecto al funcionamiento de esta técnica existe una amplia variedad tecnológica de sistemas en eyetackers, citaremos un par de ellos, de entre los otros que existen: aquellos que se colocan en la cabeza del participante y los que registran el movimiento ocular desde la distancia, que resultan mucho menos intrusivos.

Un dato curioso, además de relevante, es que cuando se explora visualmente una escena, los ojos no se mueven suavemente, sino mediante saltos o movimientos rápidos (30-120ms), a lo que se ha dado en llamar "sacadas" (J acob, 1995). Entre una sacada y la siguiente, se produce una fijación (200-600ms), periodo de relativa 
quietud del ojo que posibilita ver nítidamente la zona enfocada. El problema radica en la dificultad que estriba en detectar precisamente las "fijaciones". "No existe una técnica o medida estándar para la identificación de fijaciones, por lo que estudios que usasen medidas diferentes podrían dar lugar a resultados diferentes" (Jacob, Karn, 2003) (Poole y Ball, 2004). Se puede deducir, por ende, que tanto la técnica como la propia interpretación de los datos obtenidos pueden ser susceptibles de error.

\subsection{Imagen del Eyetracking}

Cuando aparecieron por vez primera las mediciones de "trazabilidad visual" en España, en el primer trimestre del año 2005, la página Web iprofesional.com publicó una noticia (el 18 de enero de 2005) donde se anunciaba esta técnica como "la solución para captar la mente de los cibernautas", un asunto harto complejo. En esta noticia se puede extraer una información nada desdeñable; de un lado, a quién está dirigido especialmente el conocimiento de los intereses de los usuarios y, de otro, los resultados, decía, son "claros y objetivos" acerca del comportamiento del internauta.

"Los estudios basados en la trazabilidad visual son actualmente una herramienta de interés para los medios de comunicación en la Web, los portales de Internet $y$ otros negocios online que necesitan demostrar de forma inequívoca la eficacia de la publicidad y su lectura por parte de sus navegantes",

se puede leer en la noticia. Además en ella incluye declaraciones del director gerente en aquella fecha de alt64, la consultora española pionera en Eyetracking: " $\mathrm{La}$ tecnología Eyetracking (o la trazabilidad visual) es extremadamente eficaz porque permite profundizar en el pensamiento del usuario, prever sus movimientos $y$ determinar sus zonas de mayor atención". 


\section{Terminología relacionada}

Antes de continuar avanzando sobre el tema que nos ocupa, es recomendable definir y explicar específicamente algunos conceptos estrechamente relacionados con la tecnología Eyetracking.

\section{- Usabilidad}

La usabilidad es definida como "la efectividad, eficiencia y satisfacción con la que un grupo de usuarios específicos puede realizar un conjunto específico de tareas en un ambiente particular" (Internacional Standards Organization -ISO-). Guillermo Franco, autor del libro Cómo escribir para la Web, simplifica esta definición, con un propósito pedagógico, asegurando que la usabilidad describe "la facilidad de uso":

“Qué tan fácil es trabajar para con el texto en este sitio Web?, ¿qué tan fácil es encontrar información específica? Comparado con lo que esperaba, ¿qué tan rápido hizo las tareas? Y, ¿qué tan difícil fue concentrarse en la búsqueda de información?"(Franco, 2008, p. 48).

Nielsen, experto tanto en estudios basados en el Eyetracking como en usabilidad, afirma:

"En la Web, la usabilidad es una condición necesaria para la supervivencia. Si un sitio Web es difícil de usar, la gente lo abandona. Si la página de inicio falla para establecer claramente lo que una compañía ofrece y lo que los usuarios pueden hacer en el sitio Web, lo abandonan. Si la información del sitio Web es difícil de leer o no responde a las preguntas clave de los usuarios, estos lo abandonan"(Franco, 2008, pág. 47). 
Algunas de las conclusiones, suscritas en el libro de Franco, procedentes de la labor de Nielsen demuestran que: los usuarios prefieren el lenguaje objetivo, los textos concisos y el diseño escaneable, y subraya que "la forma de estructurar los textos en Internet está determinada más por razones funcionales que por simples razones estéticas, literarias o artísticas"(Franco, 2008, pág. 48).

\section{- $\quad$ Lectura en línea}

El proyecto Eyetrack del Instituto Poynter ha estudiado los hábitos de lectura del usuario. El lector en línea es el lector-usuario que lee la pantalla de un ordenador, mientras que el lector en papel es el lector de prensa tradicional. Según sea el movimiento de los ojos sobre la pantalla o las páginas impresas, hay dos tipos de lectura:

- Los lectores "metódicos": que leen de arriba hacia abajo, sin saltar a un lugar a otro de la página, leen el texto completo y releen algunos materiales, y en línea utilizan la barra de navegación para localizar noticias.

- Los lectores que "escanean", que leen los títulos, bajadas y párrafos destacados del texto, y después saltan a las imágenes u otros elementos, sin releer.

Educar, un portal educativo argentino, se hacía eco de algunos hallazgos descubiertos por el Instituto Poynter, el 9 de marzo de 2007; en primer lugar, la tasa de lectores que leen las noticias completas es mayor entre los lectores en línea que entre los lectores en papel y, en segundo lugar, la mitad de los que leen en línea escanean la información y la otra mitad lee en forma metódica, mientras que el 75\% de los lectores en papel tienden a hacer una lectura metódica.

La investigación Eyetrack 07 describe a los lectores metódicos como aquellos que no leían de arriba hacia abajo. 
"No ojeaban con mucha frecuencia. (...) cuando se conectaban, usaban los menús descendentes y las barras de navegación para localizar historias", dice el documento final del informe. "Pudieron haber leído una parte de una historia, mirar fotos u otros ítems en el paquete, pero generalmente no regresaban una vez habían dejado el texto"(Franco, 2007, pág. 36).

Franco subraya una idea más que recomendable, que hay que estructurar los textos para el ambiente online teniendo en mente el comportamiento y las metas del usuario.

\section{- Palabras clave}

Ramón Salaverría, director del Laboratorio de Comunicación Multimedia de la Universidad de Navarra, se refiere al uso de las palabras clave dentro de un texto 0 expresiones resaltadas así: "Con las palabras clave, al tiempo que se vivifica la uniformidad del texto, se facilita al lector su rápido rastreo y la localización de nombres y expresiones de especial interés". Salaverría añade que el periodista debe saber el altísimo poder magnético que tienen las palabras claves para la mirada del lector:

"Muy probablemente usted mismo lo habrá comprobado al posar su mirada en primer lugar en estas palabras en negrita antes de comenzar a leer este párrafo por el principio. Dado este alto magnetismo, es preciso emplear este recurso con mesura; de 10 contrario, puede llegar a entorpecer la lectura reposada del texto" (Salaverría, 2005, pág. 86).

En este sentido, se puede deducir que las palabras clave están íntimamente vinculadas al estudio del Eyetracking, llaman la atención del lector y pueden romper 
fácilmente la lectura lineal del texto y, por tanto, pueden definir áreas de interés de la interfaz.

\section{- Zonas calientes versus zonas ciegas}

La atención visual en la interfaz del ordenador depende directamente de cómo se presenta la información en la pantalla del ordenador. El recorrido visual seguirá un trazado no definido, observando aquellas partes que más resalten, porque figuren en negrita o color o simplemente debido a su tamaño, dentro del sitio Web. Las empresas dedicadas a esta materia se encargan de analizar rigurosamente, gracias al uso de mapas de calor, las zonas que visualmente han sido más atendidas, zonas o puntos calientes y de mayor intensidad de atención por parte del lector, en detrimento de aquellas zonas que han pasado más desapercibidas, zonas ciegas 0 frías. Así, mediante el uso de representaciones gráficas, mapas de calor (heatmaps), se facilita al cliente final qué partes de la página Web atrae más al usuario y cómo se distribuye su interés en la misma.

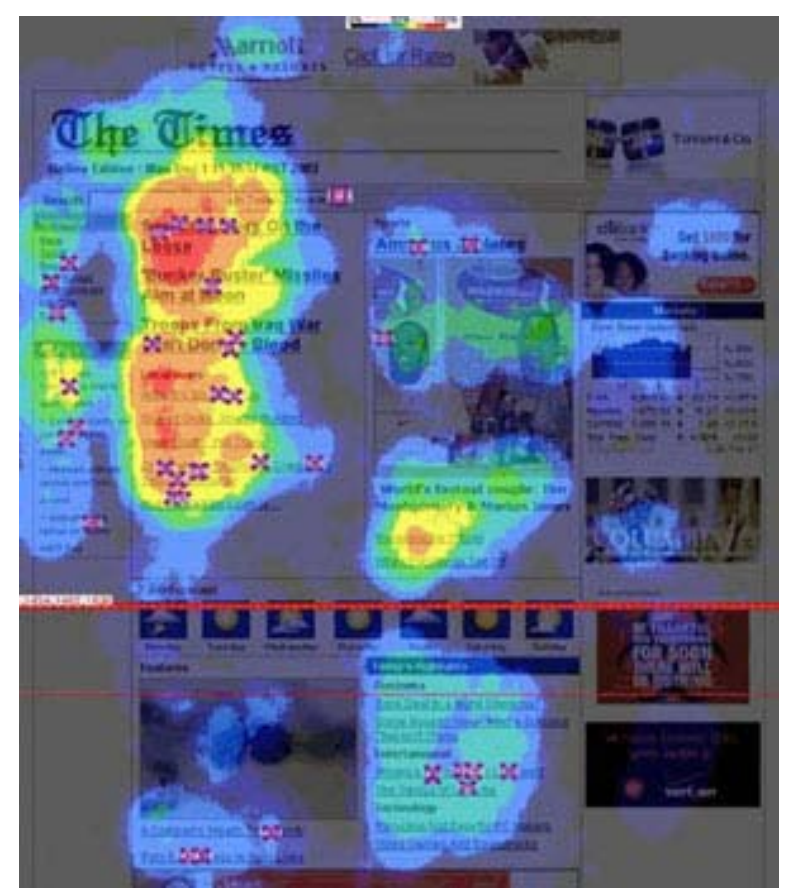


Esta foto es un ejemplo de una representación gráfica de mapa de calor, las zonas más cálidas corresponden a las más observadas, mientras que las zonas menos coloreadas pertenecen a las partes que han sido menos atendidas por el usuario. El Instituto Poynter en este estudio asegura que la navegación en la parte superior de la página digital juega con ventaja respecto a las partes inferiores, sin embargo, la gente tiende a escanear las porciones más bajas de la página en busca de algo que capture su atención. La solución que propone el Instituto Poynter al respecto versa en la necesidad de escribir titulares interesantes y atractivos y en poder resaltar las palabras clave en negrita. El Instituto Poynter considera que el usuario medio ubica el centro de atención en la parte superior izquierda (www.artvisual.net).

\section{- Patrón de lectura en F}

De acuerdo con las investigaciones de Eyetracking 07 dirigidas por Nielsen, hay un movimiento horizontal en la parte superior del área de contenido, de izquierda a derecha, luego se regresa, hay un movimiento vertical por la parte izquierda de la pantalla; hay un segundo movimiento horizontal, pero más corto que el anterior y, finalmente, un movimiento vertical en la parte izquierda de la pantalla. A continuación, se muestra una serie de mapas de calor que presentan qué zonas son las más observadas, la imagen central presenta el patrón de lectura en $\mathrm{F}$ estudiado por Nielsen.
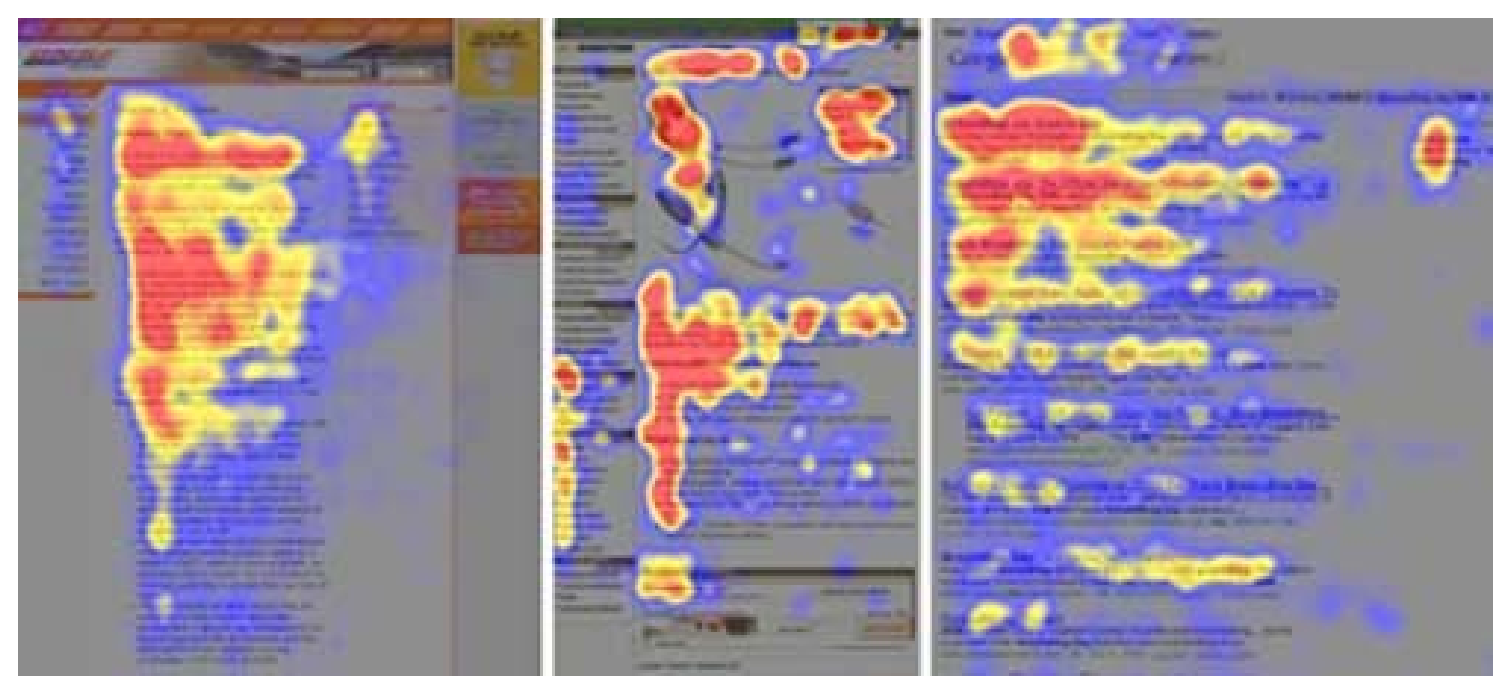


\section{Investigaciones recientes}

Parafraseando a Inma Martín Herrera, autora de ensayos sobre Ciber-redacción,
"el periodismo electrónico surge, por tanto, para dar respuesta y satisfacer las nuevas y más exigentes necesidades informativas surgidas en la Sociedad del Conocimiento. Para lograr su cometido, este nuevo periodismo cuenta con su propio canal: Internet, $y$ soporte: la pantalla de un ordenador, sus propias características, así como sus propias ventajas e inconvenientes; una serie de factores que lo convierten en un medio de comunicación diferente, y al mismo tiempo complementario, de la prensa, la radio y la televisión".

Ya se ha indicado anteriormente que el uso de la técnica del Eyetracking es una investigación relativamente reciente. Fue a partir del año 2000 cuando la Standford University y el Instituto Poynter publicaron el primer estudio acerca de las noticias en websites, de las que se extrajeron sorprendentes resultados. En primer lugar, descubrieron que los lectores de webs, lectores en línea, miran primero el texto (especialmente breves), no imágenes, un hecho que contradice el comportamiento medio de la lectura en prensa escrita, como se observó en el estudio Eyes on the News en 1991. En segundo lugar, otro hallazgo inesperado se refería a la captura de atención que recibían los banners o anuncios publicitarios en la web, cuyo tiempo de fijación tenía una media de un segundo, es decir, el suficiente tiempo para ser percibido, en contra del pensamiento predominante en ese momento. Estas dos teorías no han estado exentas de críticas.

Los mismos investigadores de Eyetrack, después de casi una década de observación y análisis, pertenecientes al Instituto Poynter, centro de investigación y educación con sede en Florida (Estados Unidos), son conscientes de que las deducciones sobre "el seguimiento del ojo" han de ser combinadas con otras medidas utilizadas por los 
sitios web de noticias: pruebas de usabilidad, sesiones de grupo (focus group) y análisis de registros ( $\log$ analysis). Al menos, así lo señalan Steve Outing y Laura Ruel en su artículo Cómo lucen los sitios Web a través de los ojos de los lectores. No obstante, han puesto de relieve algunas comprobaciones dignas de mención:

1- Los titulares dominantes atraen la atención con más frecuencia cuando se ingresa en la página, especialmente cuando se encuentran en la parte superior derecha.

2- Las tipografías más pequeñas motivan el comportamiento de visión enfocada (esto es, leer palabras), mientras que las tipografías más grandes promueven el escaneo. Según sus pruebas, la gente pasa más tiempo enfocada en la tipografía pequeña que en la grande. Igualmente sucede con los titulares, los más largos animan el escaneo más que los más cortos.

3- Si el promedio de lectura de un titular obtiene menos de un segundo en cuanto atención, proponen utilizar palabras "cautivadoras de atención" al principio del titular, sobre todo, si son largos.

4- La navegación ubicada en la parte superior de la página de inicio fue vista por el mayor porcentaje de sujetos de prueba y mirada por la más larga duración.

5- Los datos revelaron que los párrafos cortos recibían el doble de atención visual que aquellos párrafos más largos.

6- Respecto a los banners publicitarios, reciben más fijación del ojo en la medida que se encuentra en la parte superior de la página y juegan con ventaja si están próximos a la información de la Web.

7- A mayor tamaño de una imagen, el usuario se toma más tiempo para mirarla. 
8- Observaron que los participantes recordaban correctamente los hechos, nombres y lugares cuando se presentaba en formato de texto, sin embargo, la información conceptual, no familiar, era más recordada en un formato multimedia.

La mayor contradicción desprendida de los estudios de Eyetrack07 del Instituto Poynter es su proclamación de que la gente lee más y en profundidad en los sitios de prensa digital, incluso más que en los periódicos impresos, aduciendo que no supone un inconveniente la lectura en una pantalla de ordenador. El pensamiento general de muchos periodistas y expertos en medios de comunicación es la teoría opuesta. Martín Herrera lo expresa de la siguiente manera:

"En primer lugar, y en relación al soporte, cabe destacar que es más difícil y cansino leer en la pantalla de un ordenador que en el papel. Por este motivo, y también debido a la rapidez con la que el lector online pretende informarse, en Internet se tiende a acortar los textos. Se trata de sintetizar y resumir las noticias. El objetivo es describir correctamente todos los detalles importantes del acontecimiento, pero evitando redactar artículos excesivamente largos que, sin embargo, si tendrían cabida y sentido en la prensa analógica".

No obstante, Nielsen resalta la tesis de que los lectores están ahora dispuestos a leer más, sin importar el tema de la resolución de pantallas y agrega: "El comportamiento más común es cazar información y ser brutal en ignorar detalles. Pero una vez la presa ha sido cogida, los usuarios algunas veces se sumergirán más profundamente. Así, el contenido Web necesita soportar ambos aspectos del acceso a la información: "búsqueda y consumo "(Franco, 2007).

Lo cierto es que el entorno digital está ganando tantos adeptos cada día, que se está cuestionado mucho la supervivencia de la prensa escrita, trasladada con muchas más 
posibilidades en el formato online. Los diarios digitales proliferan exageradamente hasta el punto de que incluso un ciudadano medio puede crear su propio periódico, convirtiéndose en su propio editor, sin necesidad de ser periodista, como ha anunciado recientemente la Web Periodista Digital. La realidad se impone y el número de horas dedicada a Internet aumenta en detrimento del uso de otros medios de comunicación, en concreto, y como se ha explicado anteriormente, la más afectada: la prensa escrita.

Un informe de la Asociación Mundial de Periódicos lo reconoce:

"La historia de los periódicos gratuitos y de su implacable desarrollo - expone - es, de hecho, la historia de una nueva generación. Se le puede llamar la generación de Internet... Los más jóvenes no asocian la idea de lectura con la de esfuerzo. Para ellos, la lectura es puramente visual, un ejercicio similar al de ver la televisión, la pantalla de un ordenador o de un teléfono móvil".

De hecho, ya se denominan a los diarios digitales "viewpapers" (diario visual), en lugar de "newspaper" (diario de actualidad) (Fogel y Patiño, 2007, pág. 23).

\section{El caso español}

En España una empresa especializada en soluciones de Internet Intelligence y Marketing Digital, llamada alt64, introduce el Eyetracking a partir del año 2005, con la colaboración de la AIMC (Asociación para la Investigación de Medios de Comunicación). Con el objetivo de aprovechar al máximo el rendimiento de las empresas online, alt64 firma un acuerdo con Tobii Technology, empresa sueca pionera en el desarrollo de soluciones Eyetracking para estudiar el movimiento ocular. Desde entonces, alt64 ha ofrecido a las empresas un análisis completo tanto 
de la navegabilidad, usabilidad de sus páginas digitales como de la eficacia procurada para la publicidad en Internet.

Actualmente, no hay más que pinchar en su Web www.alt6.com para ponerse en contacto con ellos. La metodología empleada se divide en: patrones de comportamiento visual, visibilidad de la publicidad en Internet y la efectividad de ésta. En uno de sus estudios, publicado en noviembre del año 2005, se estudiaron seis diarios digitales: ABC, El Periódico, El Mundo, La Razón, La Vanguardia y La Verdad, y se utilizó una muestra de 17 personas. Atendiendo a los resultados del mismo, llama la atención algunas de sus comprobaciones:

1. Existe un patrón de navegación diferente en las mujeres que en los hombres, que puede verse alterado por el contenido gráfico de la página digital.

2. Por norma general, más de la mitad de los lectores fijan su vista en los anuncios publicitarios, que no está predeterminado ni por su sexo, su experiencia como internauta o por su hábito de lectura en diarios online.

3. Los banners situados en las cabeceras de las páginas Web son los más efectivos tanto en la fijación de la mirada como en la retención de la marca, aunque un rascacielos que siga al usuario mientras navega puede superar en resultado a toda la publicidad horizontal.

Se puede comprobar perfectamente que los hallazgos de alt64 no distan en absoluto de las realizadas por el equipo estadounidense.

\section{Críticas versus posibilidades}

¿Tiene sentido el estudio de nuestra mirada delante de una página Web? Desde luego. ¿Qué efectividad poseen los descubrimientos de Eyetracking en general? Pues, probablemente, sirvan como guías para obtener información que no deba ser denostada en su mayoría, a pesar de que no sea una técnica infalible, por supuesto. 
El investigador Howard Finberg, en su artículo "Eyetrack is not a solution", pone de manifiesto que el Eyetrack es una herramienta y no una solución, aunque reconoce el hecho de que para los periodistas supone de gran ayuda a la hora de realizar su trabajo digital mejor. En concreto, Finberg señala que estos estudios provocan la discusión al generar preguntas y preocupaciones relativas al entorno online y suscribe la opinión de Rusty Cotas, director de MORI Research, una compañía que trabaja en el campo de los diarios digitales:

"El desacuerdo puede ser la mejor cosa para la investigación, porque nos fuerza a buscar más fuertemente los hechos en si que las presunciones y emociones. Primero, asegúrate de que el dato es correcto. Investigar es como cocinar un pastel: hay muchos ingredientes, y todos tienen que tener que ser los correctos".

En la revista especializada No solo usabilidad se puede leer que la técnica del Eyetracking ha sido calificada como "prometedora", una etiqueta que tiene una connotación positiva y negativa al mismo tiempo. "Lo positivo es que debe tratarse realmente de una tecnología prometedora, ya que de lo contrario hubiera sido descartada a estas alturas. Lo negativo es que algo la ha mantenido estancada en esta etapa de simple "promesa". Hay varias posibles razones para esta situación, entre ellas, los problemas técnicos en los estudios de usabilidad, el trabajo intensivo de la extracción de datos, y las dificultades en la interpretación de los datos" (Jacob y Karn, 2003). Es decir, la complejidad de trabajar con el comportamiento de los internautas delimitada por la técnica del Eyetracking conlleva serios riesgos a la hora de proponer teorías concretas. A pesar de todos los avances obtenidos, aún debe ir perfilándose mucho más. 


\section{Conclusiones}

Después de este acercamiento a la herramienta del Eyetracking, cuyo fin es el estudio del movimiento ocular delante del ordenador, en concreto, del comportamiento de ojo humano ante los diferentes diseños que ofrece una página digital, se puede afirmar que, además de ser un campo emergente, es una labor que merece la pena tener en cuenta, especialmente, para quienes trabajan en el ámbito de la investigación y del periodismo.

¿Cómo suele leer el usuario o el internauta los titulares? ¿Qué tipos de lectura existen? ¿Se detiene en las palabras clave? ¿Qué niveles de atención procura hacia cierto tipo de información, según sea más larga o más breve, mejor presentada o según se encuentre su ubicación? ¿Tiene eficacia la publicidad? ¿Cómo se fomenta el impacto visual del lector en línea? Aunque el Eyetracking sólo sirva como herramienta de uso y conlleva unas limitaciones intrínsecas, ya sea por su metodología o por si técnica, sus estudios trascienden al ámbito académico por lo que fomentan un debate más que interesante.

Una vez trasladada esta discusión al entorno universitario y al profesional, se generarán los grupos de discusión pertinentes y los análisis, que a su vez, apoyen o contradigan algunos de los hallazgos obtenidos por el Instituto Poynter. En cualquier caso, y al centrarse en el comportamiento del hombre frente a Internet, no dejará de estar en auge en la medida que el increíble éxito de la web tenga el apogeo como el que hoy está teniendo. En definitiva, el Eyetracking no debería ser menospreciado, ni por supuesto considerado infalible, más bien, debería servir de referencia y guía para continuar ahondando en los efectos del medio digital en la población. 


\section{Bibliografía}

Alt64, AIMC: Estudio Eyetrack Medios España. “Análisis del comportamiento visual de los internautas y la efectividad de la publicidad online", estudio realizado por la empresa alt64, con la colaboración de AIMC, noviembre de 2005.

CEBRIÁN, J.L. (2009). El pianista en el burdel. Galaxia Gutenberg. Barcelona.

FRANCO, G. (2008). Cómo escribir para la Web. Bases para la discusión y construcción de manuales de redacción "online". Universidad de Texas. Austin.

FOGEL, J.F. Y BRUNO, P. (2007). La prensa sin Gutenberg. El periodismo en la era digital. Punto de Lectura. España.

FINBERG, H. (2004). "Eyetrack is not a solution". Disponible en: www.poynterextra.org/EYETRACK2004/solution.htm)

GRUFFAT, C. (2007). “La lectura de noticias en línea, según el último estudio del Instituto Poynter" (noticia de la Web argentina www.educ.ar), 9 de abril.

HASSAN, Y. "Eye-Tracking en Interacción Persona - Ordenador", No solo usabilidad, Revista Multidisciplinar sobre diseño e interacción (issn 1886-8592).

JACOB, R.J.K.; KARN, K.S.. Eye Tracking in Human-Computer Interaction and Usability Research: Ready to deliver the promises. Disponible en: www.cs.tufs.edu/jacob/papers/ecem.pdf

MARTIN, I.. "Ciber redacción periodística: Nuevo lenguaje para un nuevo medio". Disponible en: www.chasqui.comunica.org 
ISSN: 1576-3420 DOI: http://dx.doi.org/10.15198/seeci.2008.16.98-114

OUTING, S. Y RUEL L.. "Eyetrack: A History of News Consumer Behaviour". Disponible en: www.poynterextra.org/EYETRACK2004/history.htm

OUTING, S. Y RUEL L.. "Eyetrack III: Cómo lucen los sitios Web a través de los ojos de los lectores", artículo traducido por Guillermo Franco. Disponible en: www.poynterextra.org/EYETRACK2004/main-spanish.htm

Iprofesional.com: "El "eye tracking" se impone para medir el uso de las páginas Web", 18 de enero de 2005.

SALAVERRÍA, R. (2005). Redacción periodística en Internet. EUNSA. Navarra.

\section{WEBGRAFÍA}

www.alt64.com

www.educ.ar

www.iprofesional.com

www.nosolousabilidad.com

www.poynterextra.org

www.poynter.org 